\title{
Estudio del lenguaje desde la perspectiva sociocultural $^{*}$
}

\author{
Estudo da linguagem a partir da perspectiva sociocultural \\ Study of the language from a sociocultural perspective
}

Recibido el 22 de abril de 2017. Aceptado el 17 de julio de 2017

\author{
Julián Laboy Rodríguez** \\ Puerto Rico \\ José A. Maldonado-Martínez ${ }^{* * *}$ \\ Puerto Rico
}

- Para citar este artículo:

Laboy Rodríguez, Julián y Maldonado Martinez, José A. (diciembre, 2017). Estudio del lenguaje desde la perspectiva sociocultural. Ánfora, 24(43), 17 -

38. Universidad Autónoma de Manizales. ISSN 0121-6538.

\section{Resumen}

Objetivo: determinar los aportes de investigaciones recientes sobre el lenguaje desde la perspectiva sociocultural. Metodología: se realizó un análisis de contenido temático con base en categorías preestablecidas: lenguaje interno, tipos de enseñanza, bilingüismo, y otras de carácter emergente: juego y participación en práctica. Las bases de datos que se utilizaron para la búsqueda fueron Psych Info, EBSCO, JSTOR y RedALyC. Se seleccionaron 38 investigaciones publicadas en revistas indexadas desde 2010. Resultados: se evidenció que, desde la perspectiva sociocultural, los estudios aportan una diversidad de

\footnotetext{
* Este artículo es producto de una revisión de literatura extensa la cual fue evaluada como trabajo final de un curso graduado del Departamento de Psicología de la Universidad de Puerto Rico, Recinto de Rio Piedras. El curso se titula "El Estudio Sociocultural de la Mente". El mismo fue ofrecido de agosto a diciembre de 2016.

** Magíster. Psicólogo. Universidad de Puerto Rico, Recinto de Rio Piedras. Correo electrónico: julianlaboy@gmail.com

***Psicólogo. Universidad de Puerto Rico, Recinto de Río Piedras. Correo electrónico: jose.maldonado16@ upr.edu
} 
temas relacionados con el lenguaje como la participación en práctica, el proceso de cambio de un lenguaje dirigido a uno interno, la importancia del juego en el desarrollo, los beneficios de la enseñanza explícita y el impacto del aprendizaje de un segundo idioma. Conclusiones: se concluye que unos temas parecerán saturados, pero una teoría sociocultural apostaría a que en realidad esto es inverosímil. El ser humano es producto de distintos contextos sociales, culturales e individuales, por lo que siempre existirán miradas distintas para abordar los temas relacionados con el lenguaje.

Palabras clave: Lenguaje; Enfoque sociocultural; Efectos.

\begin{abstract}
Objective: this paper aims to determine the contributions of recent research on language from a sociocultural perspective. Methodology: a thematic content analysis was performed based on pre-established categories: internal language, teaching types, bilingualism, and other emerging categories: games and practical participation. Psych Info, EBSCO, JSTOR and RedALyC were the sources of information and 38 published research articles were selected from indexed journals since 2010. Results: from the sociocultural perspective, it was evident that, these studies contribute to a diversity of issues related to language, such as: practical participation, the process of change from a guided to an internal language, the importance of games in development, the benefits of explicit teaching, and the impact of learning a second language. Conclusions: it is concluded that some topics will seem to be studied in abundance but not from a Sociocultural Theory perspective. The human being is a product of different social, cultural and individual contexts; thus, there will always be different approaches to address issues related to language.
\end{abstract}

Keywords: Language; Sociocultural approach; Effects.

\title{
Resumo
}

Objetivo: determinar as contribuições de pesquisas recentes sobre a linguagem a partir da perspectiva sociocultural. Metodologia: uma análise de conteúdo temática foi realizada com base em categorias pré-estabelecidas: linguagem interna, tipos de educação, bilinguismo, e outras de caráter emergente: jogo e participação na prática. As bases de dados que foram utilizadas para a busca foram Psych Info, EBSCO, JSTOR e RedALyC. Foram selecionadas 38 pesquisas publicadas em revistas indexadas desde 2010. Resultados: evidenciou-se que, desde a perspectiva sociocultural, os estudos 
fornecem uma variedade de temas relacionados à linguagem, como a participação na prática, o processo de mudança de uma linguagem dirigida a uma interna, a importância do jogo no desenvolvimento, benefícios do ensino explícito e o impacto da aprendizagem de uma segunda língua. Conclusões: conclui-se que algumas questões parecem saturadas, mas uma teoria sociocultural apostaria a que na realidade isso é inverossímil. O ser humano é o resultado de diferentes contextos sociais, culturais e individuais, de modo que sempre existirão diferentes perspectivas para abordar questões relacionadas à linguagem.

Palavras-chave: Linguagem; Abordagem Sociocultural; Efeitos.

\section{Introducción}

Desde la perspectiva sociocultural el lenguaje se estudia por numerosas vías. Por ejemplo, Rodríguez (2009) se concentró en trabajar asuntos internos -o biológicos pertinentes al sujeto- y externos -sociales y culturales- y la relación entre ambos. Otros autores investigan desde prácticas que ocurren en ambientes académicos y en ámbitos externos a la academia, incluso en contextos virtuales (Ferreira, 2014; Peterson, 2012). Igualmente, Halle et al. (2014) y Valdez (2012) se enfocan en el lenguaje principal de una persona y en observar las diferencias que ocurren en quienes aprenden un segundo idioma. Por ello, esta investigación se propone conocer qué se ha aportado al conocimiento del lenguaje desde la perspectiva sociocultural.

Ahora bien, ¿Por qué enfocarse en el estudio del lenguaje relacionado con lo sociocultural? Para contestar esta interrogante, es necesario estudiar sus antecedentes, o lo que es lo mismo, mirar las aportaciones más importantes de los autores clásicos de la perspectiva sociocultural. Por un lado, Vygotski (1929) entendía que el conocimiento humano se produce desde un plano interpersonal hacia un plano intrapersonal. Esto se debe a que el individuo nace en un contexto sociocultural pre-existente y, por tanto, enfrenta el imperativo de internalizar gradualmente las actividades culturales, hasta que las mismas se transformen en actividades mentales. Por otro lado, Luria (1984) propuso que el análisis y la clasificación de objetos por medio de la palabra hacen posible el pensamiento humano. Y ello, en conjunto con su función de medio de comunicación, hace 
posible que se transmitan representaciones sensoriales y racionales a lo largo de la historia social (Luria, 1984).

Entonces, Vygotski (1994), Luria (1987) y Orrú (2012), argumentan que la mediación del lenguaje establece una distinción cualitativa entre las funciones mentales superiores de los seres humanos y las funciones mentales inferiores de las otras especies. Es decir, la planificación de la acción voluntaria mediada por el lenguaje, es una cualidad que solo se encuentra presente en la especie humana (Vygotski, 1930). Por consiguiente, es importante resaltar que todo el amplio cuerpo de conocimientos que comprende la perspectiva sociocultural, se encuentra permeado o atravesado por el papel central del lenguaje.

Además, el trabajo de Vygotski y Luria desde la perspectiva sociocultural y sobre el lenguaje se mantiene vigente. Por ejemplo, Daniels (2008), ha compilado las temáticas de las investigaciones más recientes con la perspectiva sociocultural como marco teórico. Sin embargo, los trabajos de este corte conceptualizan los procesos cognitivos en general, aplicando el lenguaje de manera un tanto indirecta. En particular, hablan de cómo el aprendizaje y la cognición de forma general se encuentran situadas y distribuidas en las personas y los materiales que usan. En otras palabras, procesos cognitivos como el lenguaje deben ser investigados más allá de un enfoque reduccionista, en el que se estudian solo en el cerebro y en los objetos físicos. Es necesario entender estos procesos en su forma integrada, es decir, considerando tanto lo individual, como las interacciones entre personas, recursos y materiales en sus contextos específicos (Daniels, 2008).

Por lo tanto, se debe señalar que el tema del lenguaje en la teoría sociocultural suele resultar abrumador para muchos investigadores, ya que recoge bastantes posibles interacciones entre personas, recursos y materiales. Es por esto que se necesitan investigaciones que alumbren un camino que parece demasiado entremezclado y complejo, pero que en realidad no tiene por qué serlo. De hecho, Vygotski (1934) expuso que es necesario seguir estudiando al lenguaje y la cultura, debido a que son los marcos de referencia que guían al ser humano en el proceso de experimentar, comunicar y entender la realidad.

Por eso, conocer qué se ha aportado en investigaciones recientes desde la perspectiva sociocultural y sobre el tema del lenguaje permite dar voz a las experiencias en diversos tipos de sociedades y culturas. Al identificar qué se ha trabajado desde esta perspectiva, será posible saber cuánto más complejos deben ser los estudios sobre el lenguaje, qué hace falta según sus limitaciones, qué temas han sido convocados para futuras investigaciones y si todo lo anterior hace justicia a la diversidad de contextos que los produce.

Además, es necesario el estudio permanente del concepto “lenguaje” porque es un tipo de sombrilla que aborda muchísimos de los temas de la teoría sociocultural 
(Luria, s. f.). Estos asuntos abarcan todo lo que distingue a los seres humanos, cómo aprenden, cómo se desarrollan, cómo lo anterior impacta en su consciencia, entre otras cosas. De hecho, el lenguaje es un tema complejo, debido a que las personas son producto de una diversidad de contextos, circunstancias y asuntos individuales que se relacionan con el lenguaje. Reconociendo cómo este incide en la formación del individuo, resulta imprescindible continuar investigando y aportando conocimiento sobre su rol en el desarrollo sociocultural de la mente.

\section{Metodología}

Para cumplir con el objetivo de la investigación se creó un listado de términos que facilitaron la revisión. Estos se basaron en un trabajo previo, no publicado, en el que se revisó la literatura existente sobre el tema. Los términos de búsqueda incluyeron palabras o frases, y sus combinaciones, relacionadas a las siguientes categorías: sociocultural, lenguaje, lenguaje interno, inglés como segundo idioma, entre otros. Las bases de datos que se utilizaron para la búsqueda fueron Psych Info, EBSCO, JSTOR y RedALyC.

Con base en lo anterior, y como criterio de inclusión, se dividió la bibliografía en teóricas y empíricas. Las primeras, fueron utilizadas como marco teórico, en específico, se accedió a fuentes primarias de la teoría sociocultural como lo es el trabajo de Vygotski, entre otros; con los trabajos empíricos, se utilizaron otros criterios: debían aparecer en revistas indexadas desde el año 2010 en adelante. Este corte temporal de la revisión se utilizó como evidencia de la influencia de la perspectiva sociocultural y su vigencia en la actualidad. Esto, porque para llamarle 'reciente' a una referencia, se suele establecer como corte temporal un periodo de cinco años a partir del comienzo del trabajo que utilizará dicha referencia. Por último, era indispensable que hablaran de manera directa sobre el impacto del lenguaje en el desarrollo humano o, como mínimo, que el tema conectara fácilmente de manera implícita con la investigación.

Los términos y sus combinaciones que fueron utilizados, como "lenguaje", "lenguaje interno", "segundo idioma", "tipos de enseñanza", "Vygotski", entre otros, fueron objeto de búsqueda en bases de datos preseleccionadas para encontrar literatura sobre el lenguaje en el desarrollo humano con influencia en la perspectiva sociocultural. La búsqueda resultó en 38 investigaciones empíricas en inglés y español. Estas investigaciones fueron organizadas con una tabla diseñada por Colón (2016). Con ella se identificó el enfoque, alcance, diseño, población, técnica de recopilación de información y análisis, hallazgos significativos, limitaciones y recomendaciones del estudio. A esto se añadió las disciplinas y los países desde donde se hizo la investigación. El propósito del 
instrumento residía en facilitar el resumen de la información de los estudios para compartir y comparar la opinión de los autores del presente estudio.

Una vez identificada y resumida la información, se pudo analizar la literatura por medio de un análisis de contenido temático; en éste, se utilizaron constructos analíticos o reglas de inferencia, para moverse del texto a las contestaciones de las preguntas de investigación (White y Marsh, 2006). Dichos constructos analíticos pueden salir de teorías o prácticas existentes, la experiencia o el conocimiento de un experto en el tema y/o de investigaciones anteriores. En esta investigación, los constructos surgen del entendimiento que poseen los autores sobre la perspectiva sociocultural. De igual manera, no se pretende informar que el "contenido" de ese análisis sea algo que se descubre en el texto físico simplemente con leerlo.

Así pues, lo que aquí se denomina como el "contenido" del análisis es una interpretación consensuada entre los autores (Harwood y Garry, 2003). Este contenido será “temático” en el sentido de que se identificarán patrones generales en la información -los “temas”-, pero no se indagará a profundidad sobre detalles específicos una vez identificados estos patrones. Esto se debe a que se preferiría que esta indagación o interpretación mucho más subjetiva del contenido sea llevada a cabo con otro tipo de método como un análisis de discurso, un análisis semiótico, entre otros.

A partir de la perspectiva sociocultural, las categorías o temas que eligieron los autores fueron aquellas que correspondieron al objeto de investigación en los trabajos analizados y que compartían en varias instancias: perspectiva sociocultural, lenguaje, lenguaje dirigido, lenguaje interno, enseñanza explícita y aprendizaje de un segundo idioma. Se puede entender a las categorías y los temas como procesos psicológicos y/o sociales básicos (Corbin y Strauss, 2008; Piñuel, 2002; White y Marsh, 2006). Estos pueden surgir de manera emergente -de manera imprevista, pero indispensable para la investigación-, razón por la que se permitió dar cuenta de categorías o temas que no fueron considerados. Esto dio paso a los temas relacionados a la participación en práctica y al juego.

\section{Resultados}

A continuación, se presentarán resultados cuantitativos y cualitativos. Se comenzará con los datos cuantitativos que fueron extraídos y organizados en la tabla tomada de Colón (2016). Estos tipos de resultados tienen implicaciones teóricas, asunto que se ha intentado cubrir en esta investigación. Se trata de implicaciones que van más allá del análisis de contenido temático, pero que 
también aportan al conocimiento de investigaciones recientes sobre el tema del lenguaje desde la perspectiva sociocultural.

A los datos cuantitativos le seguirán los hallazgos cualitativos abstraídos por medio del método de análisis de contenido temático. Mientras que los datos cuantitativos aportan aspectos descriptivos de las investigaciones con tema general del lenguaje y con un marco teórico sociocultural; la información cualitativa presenta los tópicos o temas específicos de estas investigaciones. Se ha escogido una manera deductiva de presentarlos.

Se comenzará con temas generales que aplican al desarrollo humano y los procesos cognoscitivos completos: participación en práctica y la transición del lenguaje dirigido al lenguaje interno. Como se evidencia, estos temas impactan al lenguaje indirectamente en ocasiones $y$, en otras, tratan al tema de manera mucho más directa. A estos le seguirán temas más específicos que pueden ser vistos como factores que impactan el desarrollo del lenguaje: el juego, la enseñanza explícita y el impacto del aprendizaje de un segundo idioma. Nuevamente, unos temas trabajan la temática del lenguaje de manera indirecta, mientras que otros son mucho más directos. Sin embargo, todos trabajan desde una perspectiva sociocultural, por lo que evidencian el estado actual de cómo se investiga desde esta perspectiva el tema del lenguaje y sus aportaciones en contextos particulares.

\section{Diseño de las investigaciones}

En relación con el enfoque de las 38 investigaciones, 20 (53\%) eran de corte cualitativo, $11(29 \%)$ eran cuantitativas y 7 (18\%) tenían un enfoque mixto. Esto es sumamente importante porque la investigación cualitativa facilita la profundización de fenómenos sociales. Por ejemplo, muchos trabajos recopilaron y analizaron información por medio de estudios de caso, entrevistas semiestructuradas, etnografía, entre otros. Por lo tanto, los datos no provienen de un número extenso de casos, pero los pocos que se trabajaron en el presente estudio fueron muy completos y profundos.

Por otro lado, 23 (61\%) seguían un diseño longitudinal -recogido de datos en más de una ocasión- y el restante recogió información de manera transversal -de datos recogidos en una sola ocasión-. El hecho de que la mayoría de los diseños fueran longitudinales brinda mayor evidencia sobre lo anterior relacionado con la profundidad del manejo de información analizada. Este tipo de diseño permite recoger información de cambio, algo sumamente importante al momento de contextualizar datos y de promover una perspectiva sociocultural.

Sobre la población con la que trabajaron las investigaciones $19(50 \%)$ lo hicieron con niños, 5 (13\%) con jóvenes, 12 (32\%) con adultos y 2 (5\%) con una combinación 
de niños y adultos. Es bastante común en las disciplinas de Psicología y Pedagogía la preferencia por el estudio de poblaciones de menor edad. Esto se debe al interés por el desarrollo humano y sus procesos cognitivos desde sus comienzos, lo que evidencia el cambio en éstos. Además, mucho de ese contenido se repite en las edades posteriores.

Las investigaciones que se revisaron provienen de distintos países. La mayoría (33\%) de estos se llevó a cabo en los Estados Unidos, seguido por Inglaterra e Irán ( $11 \%$ respectivamente), Rusia y África (7\% respectivamente). Además, hubo investigaciones de países como Australia, Argentina, Colombia, España, Holanda, Canadá, Corea del Sur y Japón. Se ha incluido esta información para dar cuenta del alcance geográfico de la teoría sociocultural y el corte geográfico de la búsqueda se encontró estrechamente relacionado con el idioma de las investigaciones analizadas. Aunque si bien existe todo un enfoque revisionista del trabajo de Vygotski, su teoría sociocultural y las traducciones de su trabajo aún son utilizadas (Yasnitsky y van-der Veer, 2016). De hecho, su impacto mundial es incuestionable.

Sobre el idioma de las investigaciones que se analizaron, 32 (84\%) fueron publicadas en inglés y el resto en español. Por otro lado, 12 de las investigaciones se relacionaban con el tema de un segundo idioma, en particular el inglés. Por último, y como se mencionó arriba, la mayoría de las investigaciones fueron publicadas en los Estados Unidos (33\%), seguido por Inglaterra con 11\%. Todo lo anterior presenta un sesgo en la comunidad científica internacional por el idioma del inglés como un tipo de lenguaje universal en la academia. De hecho, y para futuras investigaciones, muchas recomendaciones delimitaron como objeto de estudio tomar consciencia del diseño de las investigaciones mismas, además de conocer por qué son publicadas en ciertos idiomas, por qué con ciertas poblaciones, entre otros.

Sobre las disciplinas desde las que se trabajaron los artículos, $19(50 \%)$ se refirió al ámbito de la Educación; a esta le siguió la Psicología con 11 (29\%) investigaciones. También se analizaron trabajos del campo de las matemáticas y la biología, entre otras disciplinas. Esto demostró el alcance de la teoría sociocultural desde la academia. El trabajo de Vygotski tuvo mucho impacto en la disciplina de la Educación por la temática de la que esta se nutre, lo cual queda puesto en evidencia con los hallazgos de este estudio. La relación estrecha entre la Psicología y la Educación no es ninguna revelación extraordinaria (Vygotski, 1986).

Hasta aquí se han presentado los datos de corte descriptivo. Estos datos deben ser contrastados con el contenido de las investigaciones como tal. A continuación, se resumen los temas en común que se trabajaron en los estudios que conformaron el corpus de la presente investigación. Es menester recordar que estos evidencian el conocimiento específico que se produce desde la perspectiva sociocultural en relación con el tema del lenguaje. 


\section{Procesos psicológicos $\mathrm{y} / \mathrm{o}$ sociales}

Las investigaciones que se analizaron aquí tuvieron en común temas relacionados con procesos psicológicos y/o sociales. En esta sección, se profundizará en las categorías y/o temas que surgieron consistentemente de los estudios, tales como: la participación en práctica, el proceso de cambio de un lenguaje dirigido a uno interno, la importancia del juego en el desarrollo, los beneficios de la enseñanza explícita y el impacto de un segundo idioma. Como se mencionó previamente, lo anterior surgió de un análisis de contenido temático con unas categorías preestablecidas y otras emergentes. Se comenzará por discutir procesos generales que abarcan gran parte del desarrollo humano como la participación en práctica y la manifestación del lenguaje interno, para luego seguir con procesos más específicos como la enseñanza explícita y lo relacionado con segundos idiomas.

\section{Participación en práctica}

Como parte del estudio de procesos sociales generales y tomando aspectos teóricos de Vygotski (1934) y Wertsch (1998) han explorado cómo el proceso de cualquier práctica involucra a su vez al aprendizaje. La participación de las prácticas en nuestras comunidades provee un tipo de base de datos, de la cual las personas abstraen principios generales y construyen modelos sobre sus mundos. Las comunidades valorarán estas prácticas, lo cual permite que vuelvan a repetirse y ser reforzadas. En otras palabras, la interacción con las demás personas, además del contexto donde éstas ocurren, juegan un papel importantísimo en el aprendizaje y el desarrollo de una persona. Prácticas como la escolarización generan productos o aprendizajes específicos y la literatura consultada evidencia este aspecto.

Un buen ejemplo del tema se encuentra en un estudio de Townend y Brown (2016) sobre el entendimiento del auto-concepto en estudiantes doblemente excepcionales, casos muy pertinentes al estudio del lenguaje desde la perspectiva sociocultural. Estos estudiantes, que son dotados intelectualmente y a la vez tienen una discapacidad, parecen tener una influencia enorme de los maestros y el contexto en el que son educados. Por ejemplo, debido a lo anterior, un chico ve sus logros no como logros, sino que se concentra en pensar que se debe a sus capacidades especiales y que no merece crédito. Esto parece haberlo aprendido del discurso que repiten sus maestros a diario, lo cual luego reproduce con su auto-concepto. Esto es de suma importancia para el tema del lenguaje, ya que este se verá afectado por el tipo de situación en el que la persona se encuentre. 
Lo anterior también ha sido extrapolado a diferentes disciplinas, evidenciando las particularidades del contexto en el que practicamos-aprendemos. Por ejemplo, ha sido estudiado con las matemáticas (Gómez y Mojica, 2014; Roth y Gardener, 2012), la microbiología (Pérez, Martínez y Hoyos, 2014), la educación (Kucirkova, Messer y Sheehy, 2014), la psicología del desarrollo (Monforte y Ceballes, 2014), la psicolingüística (Gallego, Gómez y Ayllón, 2016), e incluso en la poesía de adultos (Myhill y Wilson, 2013). Todas generan prácticasaprendizajes diferentes, lo que a su vez generará estructuras de lenguaje específicas. La implicación es expresada por Pérez, Martínez y Hoyos (2014) cuando dicen que "lo más importante no es lo que enseñamos, sino lo que pasa en el escenario del juego didáctico, porque es lo que hacemos, puesto en situación de la clase, lo que permite el aprendizaje" (p. 88).

Las investigaciones parecen señalar que el aprendizaje y su relación con los diferentes contextos en los que se desenvuelven los individuos son inseparables. Esto es una tesis de carácter social y general que aplica a todo el desarrollo humano. Es, por tanto, el contexto de los cambios ocurridos en los procesos cognitivos como el aprendizaje del lenguaje. Este proceso de cambio por el que pasa el lenguaje es precisamente el tema que sigue a continuación, el cual fue estudiado por diferentes investigaciones analizadas en este estudio.

\section{Del lenguaje dirigido al lenguaje interno}

La creación de significados a través del uso de las palabras es un tema medular en la perspectiva sociocultural y es un asunto explícitamente enfocado en el lenguaje. Como dice Miller (2014), en el centro de la psicología sociocultural de Vygotski se encuentra el hecho de que los humanos se distinguen por la capacidad de crear significados por medio del uso de palabras. Además, asociar palabras con lo social, con la cultura y con las necesidades -hambre, calor, trabajo, afectodefinirá cómo el individuo las repite, además de cómo las aprende y cómo se desarrolla.

Esto es precisamente lo que se encontró en algunas investigaciones. Por ejemplo, en Hills (2012), se comparó la estructura del lenguaje dirigido a niños y adultos en su modo de asociación o repetición como método de aprendizaje de palabras. En el mismo, se demostró que el lenguaje se estructura de manera diferente cuando se dirige a aprendices del mismo que cuando se dirige a hablantes de alto conocimiento del idioma, específicamente en formas que aparentan estar correlacionadas con el aprendizaje temprano del lenguaje como: estructura asociativa, diversidad contextual, repetición y frecuencia del uso de las palabras. 
Las investigaciones también demostraron un interés, particularmente, en poblaciones de niños, en cómo ese lenguaje dirigido se representa hasta, eventualmente, ser lenguaje interno. Sobre su perspectiva sociocultural, se mencionó en distintos estudios y se confirma con los antecedentes a dicha perspectiva, que este tipo de lenguaje interno es un instrumento esencial de mediación del pensamiento y la autorregulación (Vygotski, 1934). Distintos investigadores han retomado estas ideas para darle continuidad y adaptarlo en diferentes contextos. Tal es el caso del trabajo de Day y Smith (2013), quienes estudiaron cómo el habla privada, la cual está usualmente relacionada con habilidades cognitivas, se asocia con tareas que provocan emociones. Los niños que participaron en ese estudio utilizaron el habla privada en tareas que involucran frustración y en situaciones relacionadas a coraje y tristeza. Los resultados sugieren que el habla privada está asociada con emociones específicas, siendo afectada por la tarea y la emoción provocada y que se asocia a estrategias de autorregulación.

El habla interna o privada en niños, por lo tanto, podría ser particularmente distinta si surge de contextos diversos, algo a lo que Vygotski apostaba. En la investigación anterior, las autoras trabajaron con la interacción entre madre e hijo. Desde otro punto de vista, Sawyer (2016) demuestra el impacto en el habla privada de los niños y en el dominio de la motivación en ambientes de juego. Escotto (2011) demuestra cómo ese lenguaje interno, moldeado por distintos contextos y enriquecido por interacciones sociales, puede involucrar a una otredad de forma dialógica. Mientras Sawyer encontró que el juego y la pedagogía basada en el juego durante la niñez temprana promueven el habla privada. Escotto habla del proceso por el cual los niños reproducen diálogos con otros y los representan mediante sus juguetes hasta que los interiorizan en palabras. Estas investigaciones hicieron referencia a lo que Vygotski (1934) decía del habla egocéntrica como una que se sumerge, hasta ser instrumento interno de pensamiento.

El sumergimiento del habla egocéntrica es, entonces, una tesis que parece aplicar al ser humano en general y en su desarrollo de procesos cognoscitivos en particular. Nuevamente, es un resultado que ha seguido desarrollándose en las investigaciones analizadas y que explícitamente se relaciona con el tema del lenguaje y la perspectiva sociocultural. Los factores que pueden ayudar en la transición de este habla bien podrían ser muy diversos. Dos excelentes ejemplos son el juego, tema del cual se ha adelantado algo en el párrafo anterior y la enseñanza explícita. Ambos también fueron estudiados consistentemente por las investigaciones analizadas en este estudio. A continuación se presenta un resumen de lo encontrado al respecto. 


\section{Juego}

Como se mencionó anteriormente con el trabajo de Sawyer (2016), el juego promueve el habla en niños. Esa internalización gradual del lenguaje, que a su vez estructura el mundo de la persona, es un tema importante para estudiar desde la perspectiva sociocultural. Vygotski (1934) mismo lo trabajó con la idea de que el lenguaje se torna el sistema simbólico dominante entre los cuatro a cinco años de edad al estructurar y mediatizar el desarrollo de habilidades, como por ejemplo las del juego simbólico. Esto es precisamente lo que concluyen Kirkham, Stweart y Kidd (2013), Goncalves (2014) y Nicolopoulou et al. (2015) en sus investigaciones.

Sin embargo, los autores mencionan que hay que tener en cuenta que el niño o la niña deben alcanzar una precisa estructura cerebral antes de lograr percibir los instrumentos culturales reales o los creados por el juego. El juego, entonces, es visto por las investigaciones recientes como una construcción. El niño no llega con un instrumento capaz de interpretar o traducir materiales culturales, como también mencionó Vygotski (1934). Es el lenguaje, o los significados sociales, los que llegan a regular el comportamiento. Por lo tanto, la actividad del lenguaje es un prerrequisito para la actividad del juego. Como un sistema organizado por reglas culturales, éstas se integran en la consciencia del individuo progresivamente. En fin, estas son ideas presentadas por los autores antes mencionados y que deben ser consideradas al estudiar la importancia y las limitaciones del juego, tal como son percibidas en este estudio.

\section{Enseñanza explicita}

Las investigaciones revisadas también han dejado clara la importancia de ciertos contextos como enriquecedores para el desarrollo del lenguaje, y por lo tanto, de la consciencia humana. Como se estableció previamente, el juego en los niños es uno de tales contextos. El mismo parece proveer herramientas sociales y prácticas que se traducen en cambios cualitativamente diferentes de los procesos cognoscitivos del individuo, incluyendo la estructura del lenguaje. A lo largo de su trabajo, Vygotski (1930/1934) también insistió en otros tipos de contextos con semejantes consecuencias. Un buen ejemplo de estos es el de la enseñanza explícita, otro de los temas que consistentemente apareció en las investigaciones analizadas aquí.

Así, por ejemplo, Ferreira (2014) observó un incremento en la eficacia de distintas tareas según el aumento en escolaridad. Entonces, los estilos de enseñanza explícitos también involucran contextos cognitivos o construcciones 
mentales que surgen en la interacción con otros; ejemplo de esto es el estudio de Borzone y Manrique (2010). En Ferreira (2014) es posible observar cómo el grado de escolaridad influye en el dominio de ciertos conceptos y formas de ser. Borzone y Manrique, se concentran en la contribución de un texto y la interacción de la maestra y de los niños alrededor del texto en la configuración de un contex to cognitivo. Notaron que “... la maestra y los niños logran construir a través del discurso un espacio de cognición expandida y compartida que favorece la realización de las operaciones cognitivas necesarias para la comprensión de los textos" (Borzone y Manrique, 2010, p. 81). Esto tiene mucho en común con otros trabajos recientes y relacionados con Vygotski (Daniels, 2008).

Ese cambio de contexto cognitivo socialmente compartido y mediatizado por el lenguaje tiene la implicación de impulsar un cambio en la consciencia en distintas poblaciones; por ejemplo, puede ocurrir en la niñez. De igual forma, Alzate y Peña (2010), demostraron que también es posible lograr esto por medio de enseñanzas entre iguales, inclusive tan tarde como en la universidad. En su estudio, encontraron la importancia que tenía la presencia de los pares en la educación, pero sobre todo si estos mostraban empatía por los estudiantes, una actitud de escucha atenta y utilizaban el recurso de la pregunta para suscitar la reflexión.

Además de asuntos internos en el contexto de educación explícita, las investigaciones también dieron cuenta de otros factores que podrían afectar la mediatización del lenguaje y el desarrollo en general. Así, Prevoo et al. (2012) demostraron que el estatus socioeconómico guarda una relación significativa con la cantidad de lectura que reciben los niños de sus padres y madres y la disponibilidad de libros con los que contaban las familias. Además, Castellaro y Roselli (2015) argumentaron a favor de cómo un estatus económico favorable provee un contexto lingüístico más eficiente, lo cual a su vez genera mejoras en la ejecución de tareas durante las edades de escolarización.

Retomando el estudio de Borzone y Manrique (2010), ¿cómo los niños llegan a dar con buenos textos si pertenecen a familias con un estatus económico que les limita las posibilidades de conseguirlos? Pensar en ese tipo de factores remite a la influencia marxista en el trabajo de Vygotski (Vieriesov, 2005). Se sugiere como resultado, entonces, que quienes se dediquen al estudio del lenguaje necesitan de esa mirada hacia aspectos externos como el contexto económico y otros.

En síntesis, tanto el juego como la enseñanza explícita son buenos ejemplos de factores que impactan el desarrollo humano en general y los cambios en el lenguaje de las personas en particular. Sin embargo, el humano es un ser muy complejo que puede seguir aprendiendo y, por ende, cambiando sus estructuras cognoscitivas, ya sea en su niñez como en otras edades. 
Un tema recurrente en las investigaciones analizadas fue el impacto que puede tener el aprendizaje de un segundo idioma en una persona. Lo más interesante de este tema es que aplica a personas de todas las edades, pero se tiende a estudiar con quienes ya dominan un idioma. Esto requiere de poblaciones de edades más avanzadas que las usuales dentro del campo de estudios del desarrollo humano. Por tal razón, es un tema que aporta mucha información al estudio del lenguaje desde la perspectiva sociocultural con poblaciones de mayor edad.

\section{Impacto de un segundo idioma}

Para reiterar, otro factor que podría afectar al desarrollo y el uso del lenguaje es todo lo relacionado al aprendizaje de un segundo idioma. Parece ser común trabajar este asunto desde un marco teórico sociocultural y su vinculación con el tema del lenguaje también resulta explícita. De hecho, Halle et al. (2014) desarrollaron una revisión de literatura para ver el impacto de un segundo idioma en el desarrollo social-emocional, utilizando categorías basadas en el marco sociocultural. Las autoras proponen que aunque existen variables contextuales e individuales que impactan el desarrollo, se deben realizar más estudios de manera sistemática en torno al desarrollo social-emocional, al igual que ponderar otras perspectivas teóricas y metodológicas.

A pesar de que no hay demasiada literatura en poblaciones que aprenden un primer y segundo idioma simultáneamente, como mencionan Halle et al. (2014), sí se han realizado muchos estudios sobre el aprendizaje de un segundo idioma en personas que ya tienen una base más sólida en su primer idioma. Por ejemplo, Korenev et al. (2016) estudiaron el uso del lenguaje por maestros en clases de inglés como segundo idioma, entre otros asuntos. Estos han notado que una gran cantidad de los maestros que han leído un libro e investigado sobre cómo enseñar un idioma, han cotejado y comentado trabajos escritos por los estudiantes en el idioma inglés y proveen retroalimentación a sus estudiantes luego de presentaciones, entre otros.

De igual importancia, estudiar la valoración del contexto, el uso social y las experiencias de aprendizaje compartidas como determinantes para medir el nivel del lenguaje como segundo idioma, ciertamente es una postura recurrente en la literatura científica sobre la perspectiva sociocultural y el tema del lenguaje (Yang y Kim, 2011; Wingyan, 2014). El aprendizaje de un segundo idioma afectará en contextos académicos, como lo han demostrado Korenev et al. (2016); pero su impacto no queda ahí. Esto se debe a que Ahn (2011) examinó el 
intercambio del lenguaje y las interacciones entre los aprendices de un segundo idioma fuera del contexto académico. La autora demostró que se crean espacios de alto aprendizaje durante el intercambio dialógico de la comparación de los dos idiomas entre los pares, entre otros asuntos. Por tanto, se propone estudiar los efectos del lenguaje cara a cara y el lenguaje por otros medios.

Ahora, Simeon (2016) estudió la diversidad de estrategias de escritura que utilizan los estudiantes al realizar tareas grupales en una clase de inglés como segundo idioma. La autora identificó cinco categorías de estrategias escritas que utilizaron los estudiantes durante las tareas grupales: (a) lluvia de ideas; (b) utilizar conocimiento previo; (c) utilizar la lengua materna; (d) el andamiaje de pares; y (e) utilizar el humor. Distintas investigaciones desde la teoría sociocultural ya comentan sobre las implicaciones sociales del andamiaje, entre otras estrategias, para que el lenguaje tenga efecto en los procesos de aprendizaje y desarrollo (Simeon, 2015; McNeil, 2012; Gagné, 2013; Khalili, Tahriri y Gholami, 2013).

Además, se debe mencionar que los análisis de las estrategias que utilizan los estudiantes de un segundo idioma tienden a surgir tanto de datos hablados, como también es posible sacar provecho a las fuentes electrónicas. Arshavskaya y Whitney (2014) recopilaron información de un blog dialógico entre dos personas que se comunicaban regularmente. Un análisis sociocultural de las interacciones entre la estudiante y su supervisora, demuestra que la primera avanzó desde la mediación iniciada por el otro, hacia un proceso de mediación comenzado por sí misma. Aunque se necesitaba más evidencia para confirmar que internalizó los conceptos pedagógicos, es necesario reconocer esto como una fuente de conocimiento valiosa e innovadora para la teoría sociocultural. De nuevo, diversas investigaciones recientes desde la perspectiva sociocultural, se han apoyado en la utilización de fuentes electrónicas (Sprow y Blouin, 2016; Peterson, 2012; Ryu, 2011; Juffs y Friedline, 2014).

Por otro lado, contrario a los estudios de Simeon (2016) y Arshavskaya y Whitney (2014), Valdez (2012) realizó un estudio cuantitativo para establecer si existen diferencias estadísticamente significativas en los logros de escritura entre los estudiantes que participaron en un programa bilingüe transicional $(M=1.89, D E=.57)$ y los de un programa completamente en inglés $(M=$ 2.0, $D E=.49$ ). De hecho, no se encontraron diferencias significativas entre las puntuaciones de escritura de los estudiantes de ambos programas, $t(60)=-.796$, $p=.43$. De igual forma, otros estudios cuantitativos relacionados con el estudio de un segundo idioma desde la perspectiva sociocultural, fueron realizados por Rassaei (2014) y Rahimi (2013).

Por último, unos estudios llegaron a establecer que también es posible abordar el tema del aprendizaje de un segundo idioma desde un acercamiento estrictamente teórico. Por ejemplo, Jang y Jiménez (2011) realizaron una crítica 
hacia la mirada tradicional en el aprendizaje de un segundo idioma, exponiendo que dicha perspectiva supone una visión encapsulada, debido a que solo se enfoca en los rasgos individuales de la persona.

En cambio, otros autores resaltaron la importancia de algunos factores socioculturales en el aprendizaje de un segundo idioma: (a) tensiones interraciales entre estudiantes; (b) estructuras de participación y relaciones de poder; y (c) la influencia de los trasfondos culturales en la formación de las metas y los comportamientos de los aprendices (Jang y Jiménez, 2011). De este modo, cada maestro que enseñe un segundo idioma, enfrenta el reto de construir un ambiente de enseñanza que facilite la utilización de estrategias positivas que enriquezcan de sobremanera el proceso de enseñanza-aprendizaje. Otro artículo reciente fue realizado por Compernolle y Williams (2013) y también abordó el tema desde un acercamiento estrictamente teórico.

\section{Conclusiones}

A partir de los hallazgos del estudio surge la interrogante ¿son estas todas las alternativas al estudio del lenguaje desde la perspectiva sociocultural? A esto es posible responder que algunos temas tal vez parecerían estar algo saturados, como lo relacionado al aprendizaje de un segundo idioma o el lenguaje interno. Sin embargo, desde una teoría sociocultural se sostiene que en realidad esto es inverosímil, debido a que los humanos son producto de distintos contextos sociales, culturales e individuales. Por tal motivo, se debe entender que siempre existirán nuevas perspectivas de los temas relacionados con el lenguaje.

Que existan miradas distintas de abordar los temas no resta importancia y pertinencia de sus aportaciones al conocimiento del estudio del lenguaje desde la perspectiva sociocultural. Por una parte, los temas más generales del desarrollo humano que fueron abordados -como el de la participación en práctica y la transición del lenguaje hacia uno interno- dejan claro el efecto catalítico en el aprendizaje de las prácticas específicas de cada comunidad. Esto se traduce a cambios en el modo en que los individuos expresan y piensan las diferentes situaciones. Por otra parte, los temas más específicos -el juego, la enseñanza explícita y el impacto del aprendizaje de un segundo idioma- son vías personalizadas por las mismas prácticas de cada comunidad. Entonces, existen procesos que es posible llamar universales.

Las investigaciones presentadas aquí parecen sugerir, y esto es consistente con la teoría sociocultural, una dialéctica entre el desarrollo humano específico en términos cognoscitivos y las prácticas específicas de las comunidades. Los temas presentados habrán sido compartidos entre las diferentes investigaciones y esto 
evidencia algo de consistencia, pero a su vez, estos temas fueron impactados por factores comunitarios o locales.

Quedaría pendiente, por lo tanto, saber qué ha resultado problemático de las investigaciones socioculturales que estudiaron el tema del lenguaje para saber cómo evitarlo o, como mínimo, cómo tratar de minimizar el impacto de esto. Muchas de las investigaciones muestran como limitación la escasa cantidad de personas que participaban de los estudios (Escotto, 2011; Roth y Gardener, 2012; Goncalves Teixeira, 2014; Townend y Brown, 2016; entre otros). Sin embargo, aún se puede sostener que lo que carecen en cantidad de la población lo tienen en la calidad de la profundización y la especialidad del tipo de características particulares que poseen. A pesar de lo anterior, es posible retomar las investigaciones que han sido revisadas, pero utilizando un mayor número de casos. Por otro lado, también es viable usar el mismo número de casos, pero realizando un cambio en el contexto cultural. Esto último permitiría comparar si existen diferencias significativas sobre el tema en particular según el cambio de contexto, tal como sugieren diversos estudios (Castellaro y Roselli, 2015; Gallego, Gómez y Ayllón, 2016; Kucirkova, Messer y Sheehy, 2014; Monforte y Ceballos, 2014; entre otros).

Además de los temas generales y específicos sobre el lenguaje, también resulta muy prometedora la cantidad de investigaciones cuantitativas y cualitativas en relación con su metodología principal. Es posible abordar un tema relacionado con el lenguaje desde la perspectiva sociocultural con una metodología mixta y esto podría verse como una conclusión sugerida por la manera en que los estudios analizados fueron diseñados. Algunas investigaciones lo lograron (Gagné y Parks, 2013; Juffs y Friedline, 2014; Korenev et al., 2016; Monforte y Ceballos, 2014; Myhill y Wilson, 2013; Prevoo et al., 2013) y los resultados aportaron una mirada más abarcadora y profunda. Se trata de un asunto clave para investigaciones futuras ya que la investigación mixta facilitaría el diseño de investigaciones longitudinales, otra de las sugerencias compartidas, al permitir más opciones para recopilar y analizar información. De hecho, el estudio del lenguaje desde la perspectiva sociocultural se beneficiaría de este tipo de diseños por la calidad de la información que se analizaría.

También resultó interesante la discontinuidad teórica de algunos trabajos. Algunas de las investigaciones mezclaban o ponían a prueba diferentes teorías, aunque bien daban prioridad a la sociocultural. A modo de ejemplo, la investigación de Kirkham, Stewart y Kidd (2013) comparaba las teorías influenciadas en el trabajo de Piaget sobre la representación de la cognición en dominios corporales específicos con aquellas influenciadas en Vygotski, las cuales enfatizaban en el andamiaje social de la cognición. Este tipo de contrastes no hicieron más que enriquecer las aportaciones de la teoría sociocultural y la investigación del 
lenguaje en general ya que siempre sugerían un acercamiento que tome en cuenta la mezcla de teorías que mejor se adapte a cada situación. Esto haría justicia a la diversidad cultural tan estimada por la perspectiva sociocultural.

Para concluir, a partir de las investigaciones analizadas en este estudio, es posible inferir que los factores pertinentes al análisis del lenguaje se encuentran en un constante proceso de cambio y transformación social y cultural. Desde el tema del lenguaje con perspectiva sociocultural, se debe reflexionar sobre cómo los seres humanos internalizan ideas sociales y culturales, a la vez que las ajustan a sus propios intereses y propósitos personales. Esto es algo consistente con las ideas originales de Vygotski (1934), ya que todos coinciden en que el ser humano adapta lo que le parece útil, rechaza lo que le resulta inútil y añade lo particular de sus circunstancias.

El conocimiento actual sobre el lenguaje desde la perspectiva sociocultural, por tanto, ha aportado saberes y prácticas que son de beneficio para las comunidades específicas desde las cuales fue estudiado. Estas comunidades han adquirido una voz propia debido a estas prácticas, a la vez que mantienen unos procesos cognoscitivos compartidos, debido a su condición como seres humanos. Esta es precisamente la riqueza del conocimiento producido al mantener una postura tan compleja que tome en cuenta características particulares sociales y la diversidad cultural.

\section{Referencias}

Ahn, T. Y. (2011). Second Language Learning in Language-Exchange Interactions: A Sociocultural and Discourse Analytic Study (Tesis de Doctorado). Recuperado de ProQuest (3485335).

Alzate, G.M. y Peña, L. B. (2010). La tutoría entre iguales: una modalidad para el desarrollo de la escritura en la educación superior. Universitas Psychologica, 9(1), 123-138.

Arshavskaya, E. y Whitney, A. (2014). Promoting Pre-services Second Language (L2) Teacher Learning via Narrative: A Sociocultural Perspective. Journal of Language and Research, 5(4), 731-741. doi: 10.4304/jltr.5.4

Borzone, A. y Manrique, M. (2010). El contexto cognitivo en situaciones de lectura de cuentos en un jardín de infantes. Lenguaje, 38(1), 65-93.

Castellaro, M. y Roselli, N. (2015). La colaboración verbal colaborativa en tres grupos etarios y dos contextos socioeconómicos. Revista Latinoamericana de Ciencias Sociales, Niñez y Juventud, 13(2), 879-891. doi: $10.11600 / 1692715 \times .13223160514$

Colón, S. (2016). Rúbrica para revisión de literatura. Inédito. 
Compernolle, R. y Williams, L. (2013). Sociocultural theory and second language pedagogy. Language Teaching Research, 17(3), 277-281. doi: $10.1177 / 1362168813482933$.

Corbin, J. y Strauss, A.L. (2008). Basics of qualitative research: Techniques and procedures for developing Grounded Theory. Londres, Inglaterra: Sage.

Daniels, H. (2008). Vygotsky and Research. Londres, Inglaterra: Routledge.

Day, K. L., y Smith, C. L. (2013). Understanding the role of private speech in children's emotion regulation. Early Childhood Research Quarterly, 28(2), 405-414.

Escotto, E. (2011, Enero). El lenguaje interno como discurso dialógico y polifónico: un caso. Revista CES Psicología. Recuperado de http://bdigital.ces. edu.co/ojs/index.php/psicologia

Ferreira, P. (2014). Vygotsky and Piaget: Scientific concepts. Psychology in Russia: State of the Art, 7(3), 24-34. doi:10.11621/pir.2014.0303

Gagné, N y Parks, S. (2013). Cooperative learning tasks in a Grade 6 intensive ESL class: Role of scaffolding. Language Teaching Research, 17(2), 188-209. doi: $10.1177 / 1362168812460818$.

Gallego, L., Gómez, I. y Ayllón, M. (2016). Eficacia de un programa de desarrollo del habla en niños con trastorno fonológico. Revista Complutense de Educación, 27(2), 805-826. doi: 10.5209/rev_RCED.2016.v27.n2.48369

Gómez, J. y Mojica, J. (2014). Una mirada sociocultural del pensamiento algebraico desde la teoría cultural de la objetivación. Revista Latinoamericana de Etnomatemática, 7(2), 81-99.

Goncalves-Teixeira, R. (2014). Reaching conversation through play: A qualitative change of activity. Psychology in Russia: State of the art, 7(3), 114-125. doi: $10.11621 /$ pir.2014.0310

Harwood, T. y Garry, T. (2003). An overview of content analysis. The Marketing Review, 3, 479-498.

Halle, T. G., Whittaker, J. V., Zepeda, M., Rothenberg, L., Anderson, R., Daneri, P. y Buysse, V. (2014). The social-emotional development of dual language learners: Looking back at existing research and moving forward with purpose. Early Childhood Research Quarterly, 29(4), 734-749.

Hills, T. (2012). The company that words keep: comparing the statistical structure of child- versus adult-directed language. Journal of Child Language, $40(03), 586-604$.

Jang, E. y Jiménez, R. (2011). A Sociocultural Perspective on Second Language Learner Strategies: Focus on the Impact of Social Context. Theory Into Practice, 50(1), 141-148. doi: 10.1080/00405841.2011.558443

Juffs, A. y Friedline, B. (2014). Sociocultural influences on the use of a web-based tool for learning English vocabulary. System, 42(1), 48-59. doi: 10.1016/j. system.2013.10.015. 
Khalili, M., Tahriri, A. y Gholami, P. (2013). The Impact of Peer Scaffolding through Process Approach on EFL Learners' Academic Writing Fluency. Theory and Practice in Language Studies, 3(10), 1893-1901. doi: 10.4304/ tpls.3.10.

Kirkham, J., Stewart, A. y Kidd, E. (2013). Concurrent and longitudinal relationships between development in graphic, language, and symbolic play domains from the fourth to the fifth year. Infant and Child Development, 22, 297-319. doi: 10.1002/icd.1786

Korenev, A., Westbrook, C., Merry, Y. y Ershova, T. (2016) 'Language Teachers' Target Language project: Language for specific purposes of language teaching. Language Teaching, 49(1), 146-148.

Kucirkova, N., Messer, D. y Sheehy, K. (2014). The effects of personalization on young children's spontaneous speech during shared book reading. Journal of Pragmatics, 71, 45-55. doi: 10.1016/j.pragma.2014.07.007

Luria, A. (1984). El papel del lenguaje en los procesos psíquicos. En Conciencia y Lenguaje (pp. 104-121). Buenos Aires: Aprendizaje Visor.

Luria, A. (s.f.). La actividad consciente del hombre y sus raíces socio-históricas. Recuperado de http://www.infoamerica.org/teoria_articulos/luriao1. $\mathrm{htm}$

McNeil, L. (2012). Using talk to scaffold referential questions for English Language learners. Teaching and Teacher Education, 28(1), 296-404. doi: $10.1016 /$ j.tate.2011.11.005.

Miller, R. (2014). Introducing Vygotsky's cultural-historical psychology. En The Cambridge Handbook of Cultural-Historical Psychology (pp. 9-46). Cambridge University Press. Cambridge Books Online. Cambridge University Press. http://dx.doi.org/10.1017/CBO9781139028097.003

Monforte, M. y Ceballos, I. (2014). Modelo de análisis de estructuras narrativas infantiles en niños de 3-4 años. Didáctica. Lengua y Literatura, 26, 329-360. doi: 10.5209/rev_DIDA.2014.v26.46843

Myhill, D. y Wilson, A. (2013). Playing it safe: Teachers' views of creativity in poetry writing. Thinking Skills and Creativity, 10, 101-111. doi: 10.1016/j. tsc.2013.07.002

Nicolopoulou, A., Schnabel, K., Ilgaz, H., Brockmeyer, C. y deSá, A. (2015). Using narrative- and play-based activity to promote low-income preschoolers' oral language, emergent literacy, and social competence. Early Childhood Research Quarterly, 31, 147-162. Doi: 10.1016/j.ecresq.2015.01.006

Orrú, S. (2012). Bases conceptuales del enfoque histórico-cultural para la comprensión del lenguaje. Estudios pedagógicos, 2(1), 337-353.

Pérez, R., Martínez, C. y Hoyos, A. (2014). Recursos didácticos para la enseñanza de la microbiología. Una propuesta para la formación docente. Praxis, $10,78-90$. 
Peterson, M. (2012). Learner interaction in a massively multiplayer online role playing game (MMORPG): A sociocultural discourse analysis. ReCALL, 24(3), 361-380. doi: 10.1017/S0958344012000195.

Piñuel, J.L. (2002). Epistemología, metodología y técnicas del análisis de contenido. Estudios de Sociolingüistica, 3(1), 1-42.

Prevoo, M., Malda, M., Mesman, J., Emmen, R., Yeniad, N., Van Ijzendoorn, M. y Linting, M. (2013). Predicting ethnic minority children's vocabulary from socioeconomic status, maternal language and home reading input: different pathways for host and ethnic language. Journal of child language, 41(05), 963-984.

Rahimi, M. (2013). Is training student reviewers worth its while? A study of how training influences the quality of students' feedback and writing. Language Teaching Research, 17(1), 67-89. doi: 10.1177/1362168812459151.

Rassaei, E. (2014). Scaffolded Feedbacks, Recasts and L2 Development: A Sociocultural Perspective. The Modern Language Journal, 98(1), 417-431. doi: 10.1111/j.1540-4781.2014.12060.x.

Rodríguez, W. (2009). La relación de las funciones ejecutivas y el lenguaje. Perspectivas Psicológicas, 3(1), 43-50.

Roth, W-M. y Gardener, R. (2012). "They're going to explain to us what makes a cube a cube?" Geometrical properties as contingent achievement of sequentially ordered child-centered mathematics lessons. Mathematics Education Research Journal, 24, 323-346. doi: 10.1007/s 13394-012-0044-5

Ryu, D. (2011). Non-Native English Speakers' Multiliteracy Learning in Beyond-Game Culture: A Sociocultural Study. MERLOT Journal of Online Learning and Teaching, 7(2), 231-243.

Sawyer, J. (2016). I think I can: Preschoolers' private speech and motivation in playful versus non-playful contexts. Early Childhood Research Quarterly, 38 (1), 84-96.

Simeon, J. (2016). Learner writing strategies of Seychellois ESL (English as a second language) secondary school students: A sociocultural theory perspective. Learning, Culture and Social Interaction, 1(1), 1-11. doi: 10.1016/j. lcsi.2015.11.002.

Simeon, J. (2015). Scaffolding Learner Writing Strategies in an ESL Secondary Class in the

Seychelles: A Sociocultural Perspective. New Zealand Studies in Applied Linguistics, 21(1), 21-38.

Sprow, K. y Blouin, D. (2016). Fostering Transformative Learning in an Online ESL Professional Development Program for K-12 Teachers. The Qualitative Report, 21(4), 781-797. 
Townend, E. y Brown, R. (2016). Exploring a sociocultural approach to understanding academic self-concept in twice-exceptional students. International Journal of Educational Research, 80, 15-24. doi: 10.1016/j.ijer.2016.07.006

Valdez, A. (2012). The Impact of Writing of Two Bilingual Education Models for English Language Learners (disertación doctoral). Walden University, USA.

Vieriesov, N. (2005). Aspectos marxistas y no marxistas de la psicología histórico-cultural de L. S. Vygotski. Outlines, 1, Recuperado de http://ojs.statsbiblioteket.dk/index.php/outlines/article/viewFile/2110/1873

Vygotski, L. (1934). Pensamiento y lenguaje. Recuperado de http://www.marxists.org/archive/vygotsky/works/words/index.htm

Vygotski, L. (1930). Instrumento y símbolo en el desarrollo del niño. En El desarrollo de los procesos psicológicos superiores (pp. 39-56). Barcelona: Grijalbo.

Vygotski, L. (1929). El problema del desarrollo cultural. Recuperado de http:// www.marxists.org/archive/vygotsky/works/1929/cultural_development.htm

Vygotski, L. (1986). Psicología y pedagogía. Ediciones Akal: España.

Wertsch, J.V. (1998). Mind as action. Oxford, New York: Oxford University Press.

Wingyan, S. (2014). Chinese L2 literacy practices: material and sociocultural considerations. Language and Education, 28(3), 237-250. doi: 10.1080/09500782.2013.819010.

White, M. y Marsh, E. (2006). Content analysis: A flexible methodology. Library Trends, 55(1), 22-45.

Yang, J. y Kim, T. (2011). Sociocultural analysis of second language learner beliefs: A qualitative case study of two study-abroad ESL learners. System, 39(1), 325-334. doi: 10.1016/j.system.2011.07.005.

Yasnitsky, A. y van-der Veer, R. (Ed.)(2016). Revisionist Revolution in Vygotsky Studies. Londres: Routledge. 